\title{
Assessment of Understanding the Concept of Motion Mechanics Using Multi Representation
}

\author{
Wawan Bunawan $^{1}$, Sabani ${ }^{2}$, Sahyar $^{3}$ \\ \{wawanbunawan@unimed.ac.id; sabani@unimed.ac.id; sahyarpasca@gmail.com\} \\ Physics Education, State University of Medan ${ }^{1,2,3}$
}

\begin{abstract}
Assessment of understanding the concept of physics is done using a three-level multiple choice test. The aim is to measure understanding of the concept, the reasons underlying the determination of the measured concept and the confidence in confirming the relationship between the truth of concept knowledge and reason for answering. The construct of the item involves multi representations (verbal, formal, graphic, and numeric) by connecting two representations in one item. The development of the problem considers three validities, namely content, construct and user validity. Test reliability uses the Cronbach alpha formula. The results showed that the content validity considered by the validator had fulfilled the validity requirements according to the proposed method. Content validity reviews the correlation between the concept content of one representation and other relevant or interrelated concept representations. Validity from the user considers the empirical understanding between the representation of concept components assessed and the representation of reasons relevant to improvements in terms of language and content correlation understanding. Data from each item can be used to trace whether there is a misunderstanding concept or the presence of alternative concepts
\end{abstract}

Keywords: assessment, concept of motion, multi representation.

\section{Introduction}

Critical Book Review (CBR), one of the mandatory tasks that will be very supportive of graduates' competencies seen in terms of cognitive mastery in the scientific field and the biggest contributor in the assessment rubric in the form of cognitive tests weighing $65 \%$ of the overall assessment. CBR has its own assessment rubric that has been developed in a holistic scale, as a more detailed complementary need to develop a Conceptual Assessment Tool (CAT) which aims to look more deeply at the mastery of concepts learned in a course as a complement of CBR.

The depth of CAT as a test instrument that will be developed can be described briefly as an indication of the consensus of the achievement of learning goals and research on the difficulties faced by students, and can be used as a measure of the effectiveness of pedagogical transformation and as an indication of students' ability to think in content material content taught (Baily et al. 2017).

The CAT development will refer to the learning objectives and achievements that have been set in the college contract, and the instructional learning of the basic courses and examine the difficulties commonly found in students towards the conceptual mastery of the material being taught. The CAT test was developed based on 5 types of representations 
(mathematical, formal, pictorial, graphic, and verbal) for each research study topic (Klein et al, 2017), this was done to describe comprehensively the understanding of concepts assessed.

The purpose of developing a multi-representation test for general physics is to determine the mastery of the concepts of test participants, trace the presence of alternative concepts or the existence of conceptual errors, and find the reasons underlying the understanding of a concept. Efforts to improve learning can be found from the reasoning for each item that deviates from the answers given based on the high and low value of the answer. Representative competence can be interpreted as the ability to understand a concept and the relationship between verbal, formal, graphical and numerical representations that interact with each other to interpret and reconstruct concepts(Ainsworth, 1999).

This paper presents three stages of developing assessment instruments. The first stage presents the role of Lecturer Field of Study (LFS) and the development plan for the items to measure mastery of the concept, the reason behind the answer statement and the level of confidence provides answers in accordance with the learning objectives. The second stage determines theoretical validity (content validity, construct validity and user validity) and test reliability. The third stage determines the detection of misconceptions based on the analysis of the results of the items or the existence of misconceptions, and finds the reasons underlying the understanding of an understanding. Efforts to improve learning can be found from the reasoning for each item that deviates from the answers given based on the high and low value of the answer. Study participants were under graduate students who took general physics courses at Faculty Mathematic and Natural Science of State University of Medan in the early years of lectures.

\section{Method and development}

\subsection{Role of lecturer field of study and objective of learning}

The success of mastering the scientific field of physics education starts from the most basic is the mastery of a concept by involving connectivity between one representation with another representation in one concept that is studied. Physics learning should be done by involving an analysis of the difficulties commonly encountered in learning physics. Development of the Concept Assessment Tool for motion material exploring concept understanding includes verbal analysis of velocity, acceleration, and particle position, formal mathematical analysis, graphical analysis, and numerical applied to regular straight motion with constant velocity, straight motion changes regularly constant velocity, bullet motion (trajectory), and Newton's law.

One of the roles of LFS assessment and evaluation examines information on cognitive abilities in mastering basic concepts through information that can be collected using assessment instruments in the form of ability tests. This field of study can be carried out through the LFS research funded by the State University of Medan. Information obtained through interpretation of test data is used to provide input on the implementation of the lecture process in the future.

The aim of general physics learning is to provide basic scientific studies of physics to review concepts or verify the existence of a physics concept through discovery, inquiry and scientific and problem solving approaches. The existence of a conceptual knowledge test provides information that is able to link learning outcomes that have been achieved with the 
successful use of a learning model or approach that is carried out and provide improvement suggestions.

\subsection{Design of development validity and reliability of test}

Development of CAT involves content, construct and user validity. Construct validity is intended to develop instruments based on theory of objective of learning which states that conceptual knowledge can be divided into verbal representations (rv), formal (rf), graphic (rg), and numeric (rn) and the connectivity between these representations ( $r v \leftrightarrow r f \leftrightarrow r g \leftrightarrow r n$ ). Content validity examines development of CAT in terms of the linkage of content based on scientific studies in physics. User validity discusses the use of CAT from the side of the user or test participant to review the logic of physics and the logic of the presentation language of the item(Sadaghiani\& Pollock, 2015).

Reliability of CAT concentrates whether the information produced will be the same when developed with the future, in other words whether the CAT test provides the same information reliability based on the time of use. The empirical formula to determine internal reliability is used by Cronbach alpha, a statistical technique to determine internal consistency which is formulated as:

$$
\alpha=\left(\frac{k}{k-1}\right)\left(1-\sum_{i=1}^{k} \frac{\sigma_{k}^{2}}{\sigma_{t}^{2}}\right)
$$

Where $\mathrm{k}$ is the number of items, $\sigma_{-} \mathrm{t}^{\wedge}(2)$ is the total variance of the test and, $\sigma_{-} \mathrm{k}^{\wedge}(2)$ is the variance of item $\mathrm{k}$. The $\alpha$ value interval is from 0 to 1 , the greater the alpha value, the higher the consistency of internal reliability.

\subsection{Detection of misconception based on item analyze}

The appearance of the three-tier multiple-choice test items can be seen in Figure 1. Each item will measure the mastery of a concept from the content of the material assessed, then followed by a follow-up question to check understanding which aims to find an explanation of the concept assessed, then the students are asked to measure individually to the correctness of the answers given.

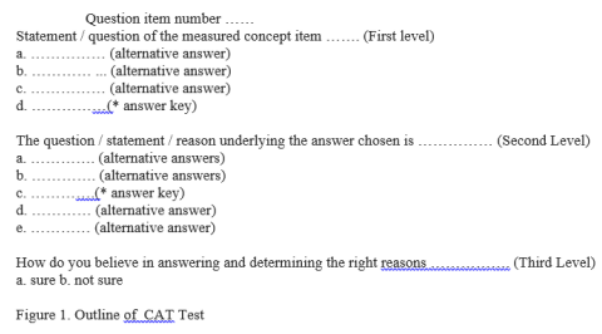




\section{Result and discussion}

\subsection{Result}

\subsubsection{Validity of test}

The source of information validity in developing a test can be traced from 5 sources related to the purpose of the test (Sireci, 2013). The method of tracking sources of validity of CAT test can be explained based on the test objectives.

The purpose of the CAT test is to measure cognitive learning outcomes, the sources of evidence of validity must meet the feasibility of the content, constructs, user responses, consequences of tests and relationships with other variables such as summative tests.

The purpose of the CAT test is to provide information to improve the learning process so that the sources of validity must fulfill the feasibility of the content, constructs, user responses, consequences of tests and relationships with other variables such as formative tests(Waldripet al,2010).

The purpose of the test is to be able to detect difficulties in mastering a concept so that the sources of validity must fulfill the feasibility of the content, constructs, user responses, consequences of tests and relationships with other variables such as tests for metacognition knowledge (Arslan et al, 2012). Based on the three objectives of test development, the CAT test has met the tracking of sources of validity in the development of the intended test.

Empirical evidence of CAT test validity based on the purpose of test development is analyzed and decided based on the consideration of the peer validator. Qualitative analysis is done to improve the quality of the editorial item, the relationship between one representation and another and the function of the choice of answers in the same item.

The peer validators requested gave scientific considerations. They are asked to give consideration by giving a check mark on indicators of sources of validity with a scale of 1-5 (not appropriate; less precise, precise, quite precise, very appropriate).Empirical validity between validators is stated with correlation index $\left(\mathrm{r}_{1-2}=0.81 ; \mathrm{r}_{1-3}=0.85 ; \mathrm{r}_{2-3}=0.82\right)$.

\subsubsection{Reliability of test}

The reliability of the CAT test to produce information between one time and the next is expressed by two methods, namely internal reliability with the Cronbach alpha method and repeated tests. Internal reliability is done to give an empirical picture for a limited scale using the Cronbach alpha formula, the test value gives a value of 0.75 . Repetition test method produces a reliability value of 0.80 using statistical correlation method.

\subsubsection{Characteristic of item test}

Information that can be obtained from a test can be traced from item characteristics. The characteristics of the items observed to provide information as an illustration of the achievement of a test goal. It can be found from the level of difficulty and differentiation of the item. The next information is the function of distractor (not the answer key) which shows the presence of alternative concepts or the existence of misconceptions.

The difficulty level of CAT test items is between the interval values $0.2-0.7$. The lowest interval value states that the achievement of the test participants in understanding a concept that was asked or confirmed in the item was only able to be answered by $20 \%$ of the test 
participants. The concept in the question is categorized as a concept that is difficult to understand by the test participants. Referring to this learning process gives an indication that the learning process needs to get attention to improving the quality of the learning process. Based on the perspective of detecting the possibility of misconceptions it provides a great opportunity for possible difficulties in mastering the concept and its relationship with other representations.

The difficulty index 0.7 provides an illustration that the mastery of concepts achieved by CAT test participants $70 \%$ has mastered the concept in question. This provides an illustration that the learning process has achieved its objectives and can be declared successful, although there are still misconceptions of the test participants. For the purpose of improving this learning process it still requires an effort to improve the quality of learning.

\subsection{Discussion}

The CAT test developed for the concept of motion is 10 questions with the category of conceptual knowledge involving the process of thinking dimensions of application, analysis and synthesis between one representation associated with other representations. Figure 2 shows one of the items from the conceptual test that links graph representation with numerical or mathematical representation.

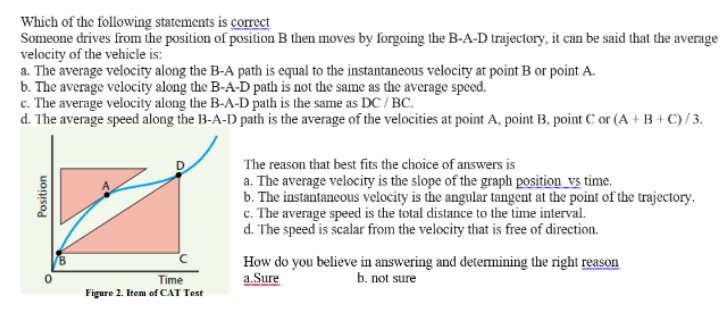

In the first part of the test, participants are required to determine the average velocity owned by a car that passes BAD trajectory. In the picture can be seen the position of the car's function of time, and can also determine the instantaneous velocity in the intended chart in accordance with the position of $\mathrm{BAD}$. If the test participants were able to determine the difference between the concept of average velocity and instantaneous velocity, it would be able to determine the average velocity based on the graph position function of the time.

The second level is checking whether the answers given are based on the right analysis or just guesses. If the test participants were able to determine the reasons correctly, it was said that the test participants were able to master the concept of average velocity from the graph position function of the time. If between the first level and the second level there is one that is answered incorrectly, there is a misconception.

At the third level the test participants are expected to be able to assess their abilities. Confidence determines understanding of the concept and underlying reasons. If at the first and second levels the response is correct and the belief about answers it at a sure level, it is said to understand the concept as a comprehensively. This item has a difficulty level index of 0.5 and a differential of 0.3 . It is said that $50 \%$ of participants master the concept and the rest have not mastered the concept of position graphs with time. The difference between high score groups and low scores that can answer correctly reaches $30 \%$ of the total number of these two groups. 


\section{Conclusion}

A three-level multiple choice test tool has been produced to measure the conceptual knowledge of mechanical motion of 30 items. Test information is collected and analyzed to determine the ability to master concepts, detect learning difficulties and conceptual errors, and improve the quality of learning.Analysis is applied to grain characteristics which have a low difficulty index value (difficult category) and have a low discriminating power index.

Acknowledgments. This work has been supported by a grant from the

State University of Medan

\section{References}

[1]Ainsworth, S. (1999). The functions of multiple representations. Computers \& Education, 33, 131-152.

[2]Arslan, H.O., Cigdemoglu, C., \& Moseley, C. (2012). AThree-Tier Diagnostic Test to Assess Pre-Service Teachers' Misconceptions about Global Warming, Greenhouse Effect, Ozone Layer Depletion, and Acid Rain. International Journal of Science Education Vol. 34, No. 11, July 2012, pp. $1667-1686$.

[3]Baily, C., Ryan, Q.X., Astolfi, C., and Pollock, S.J. (2017). Conceptual assessment tool for advanced undergraduate electrodynamics. Physical Review Physics Education Research 13, 020113.
[4]Bunawan,
W., Setiawan,
A., Rusli,
A., danNahadi.
(2015).

PenilaianPemahamanRepresentasiGrafikMateriOptikaGeometriMenggunakanTesDiagnostik.

Cakrawala Pendidikan, 34, No. 2.

[5]Dick,W., Carey, L., and Carey, J.O. (2009). The Systematic Design of InstructionBoston: Pearson

[6]Klein, P., Müller, A., \& Kuhn, J. (2017). Assessment Of Representational Competence In Kinematics. Physical Review Physics Education Research 13, 010132

[7]Motlan \& Sinuraya, J.B. (2015). FisikaUmum 1 dan 2. Medan: Larispa.

[8]Lawshe, C. H. (1975). “ A quantitative approach to content validity”. Personnel Psychology, 28, 563-575.

[9]National Research Council. (2001).Knowing what students knows: the science and design of educational assessment. Washington, DC: National Academy Press.

[10]Sadaghiani, H.R., \& Pollock, S.J. (2015). Quantum mechanics concept assessment: Development and validation study. Physical Review Special Topics - Physics Education Research 11, 010110

[11]Somov, A.: Wildfire safety with wireless sensor networks. EAI Endorsed Transactions on Ambient Systems. pp. 1-11 (2011)Sireci, S.G., (2013). Agreeing on Validity Arguments. Journal of Educational Measurement. Vol. 50, No. 1, pp. 99-104

[12]Waldrip, B \&Prain, V., \&Carolan, J. (2010). Using Multi-Modal Representations to Improve Learning in Junior Secondary Science. Res SciEduc (2010) 40:65-80 